\title{
Approximation of electro-optical characteristics of ChLC at transitions from the homeotropic texture
}

\author{
A. Rybalochka, M. Chumachkova, V. Sorokin \\ Institute of Semiconductor Physics, NAS of Ukraine, 45 prospect Nauky, 03028 Kyiv, Ukraine
}

\begin{abstract}
In this paper a method for an approximation of electro-optical characteristics of a cholesteric liquid crystal (ChLC) that describe behavior of a cholesteric material at transitions from the field induced homeotropic texture to the stable planar and focal conic textures have been proposed. To obtain the complete information about such behavior of a cholesteric liquid crystal a protracted experimental research is required. Proposed method allows to obtain all necessary approximated electro-optical characteristics of a cholesteric liquid crystal on the basis of experimental measuring only of four special electro-optical characteristics.
\end{abstract}

Keywords: cholesteric liquid crystal, homeotropic texture, liquid crystal display.

Paper received 07.05.03; accepted for publication 17.06.03.

\section{Introduction}

Bistable reflective cholesteric liquid crystal displays attract the researcher's interest because of their excellent readability, wide viewing angles, high brightness, low manufacturing cost and low power consumption with image memory. In these displays the two main unique properties of a cholesteric liquid crystal are used: bistability and Bragg reflection for light in visible spectral range [1]. Bistability of a ChLC is the presence of two stable textures of such liquid crystal materials at zero external electric and magnetic fields: the reflecting planar texture and the scattering focal conic textures. In the planar texture, the helical axes are parallel to the display cell surface normal direction and the display Bragg reflects a light peaked at the wavelength given by $\lambda_{0}=$ $=n P_{0}$, where $P_{0}$ is the pitch of the liquid crystal helix and $n$ is the average reflective index. In the focal conic texture, the helical axes are oriented randomly and incident light is weakly scattered and absorbed by black background of the display cell. Thereby display pixels with a ChLC in the planar texture look bright and in the focal conic textures look black. At the addressing of a cholesteric liquid crystal display (ChLCD) transitions between these two stable textures of a ChLC are realized.
The ways of transitions between the stable planar and the stable focal conic textures are well known [2]. A low voltage pulse can realize the transition from the planar texture to the focal conic texture. The transition from the focal conic texture to the planar texture is more complicated and can be realized only through the homeotropic texture of ChLC. In this texture a helical structure of a ChLC is unwound and all liquid crystal molecules are aligned parallel to external electric field. It can be achieved (for a ChLC with $\Delta \varepsilon>0$ ) by applying of external electric field that exceeds the threshold value $E_{C}=\left(\pi^{2} / P_{0}\right) \sqrt{K_{22} /\left(\varepsilon_{0} \times \Delta \varepsilon\right)}$, where $K_{22}$ - is a twist elastic constant, $\varepsilon_{0}$ - is a dielectric constant and $\Delta \varepsilon$-is a dielectric anisotropy. At the absence of the external electric field the homeotropic texture is unstable. Final stable texture of ChLC obtained from homeotropic texture depends on how the external electric field is turned off [3]. If the electric field is turned off slowly a ChLC transforms to the stable focal conic texture. If the electric field is turned off quickly a ChLC transforms from the homeotropic texture to the stable planar texture. It was studied that the transition of a ChLC from the homeotropic texture to the stable planar texture occurs through the transient planar texture with the pitch of the ChLC $P^{*} \approx 2 P_{0}$ 


\section{A. Rybalochka et al.: Approximation of electro-optical characteristics ...}

$\left(P_{0}{ }^{*}=\left(K_{33} / K_{22}\right) \times P_{0}\right)$. The homeotropic-transient planar transition $\left(H-P_{0}{ }^{*}\right.$ - transition $)$ is very quick and its duration for different $\mathrm{ChLC}$ can be from hundreds of microseconds to milliseconds. There is the range of voltage levels where the field induced homeotropic texture is hold and the transient planar texture is transformed to the focal conic texture by the same voltage level. These specific properties of a ChLC are used in the number of the dynamic drive schemes for bistable cholesteric liquid crystal displays [4-7].

To choose a ChLC material for real cholesteric displays a number of technical parameters of a display cell must be taken into account. There are the display cell gap, the deviation of the display cell gap on the whole area of a display cell, maximal driving voltage, an information content of a display (for passive matrix addressing - a number of columns of a display) and so on. The temperature condition of using of a ChLCD, an addressing method and necessary addressing speed are also important parameters for a determination of requirements to a ChLC material. Thus for the determination of all parameters and characteristics necessary for the right choice of a ChLC material for a ChLCD a profound experimental study of physical properties and electro-optical characteristics of a ChLC must be performed. Therefore a search of ways for a decreasing of the duration of such experimental study is a very actual and important problem.

This is our first work devoted to decision of abovementioned problem in the supposed series of papers. A method of an approximation of electro-optical characteristics of a ChLC when a cholesteric material is initially in the field induced homeotropic texture is proposed here. Furthermore this method is based on experimental measurement of four special electro-optical characteristics of investigated cholesteric material. All necessary parameters for an approximation are obtained only from these characteristics and knowledge of values of different physical parameters of a ChLC is not necessary.

We performed all our measurements on the display cell filled with the liquid crystal mixture BL126 (Merck), cell thickness $d=4.4 \mu \mathrm{m}$ at room temperature. Display Measuring System SV-200 was used for measuring of all experimental characteristics.

\section{Experiment}

First of all we perform experimental measurement of electro-optical characteristics of the ChLC. For that the sequences of voltage pulses with the waveform that is demonstrated on Fig. 1 are applied to the display cell. At the beginning a high preparation voltage $U_{p r}$ is applied to the display cell during the preparation time $T_{p r}$ to transform the ChLC into the homeotropic texture irrespective of it initial texture. Than the bias voltage pulse $\left(U_{b}, T_{b}\right)$ is applied for studying of the response of the cholesteric material. The final reflectance of the $\mathrm{ChLC}$ is measured in one second ( $\left.T_{\text {pause }}\right)$ after the bias pulse at the absence

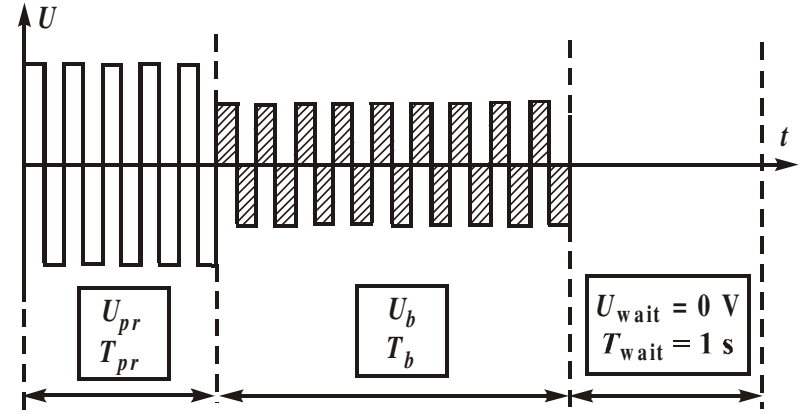

Fig. 1. The voltage waveform to measure the electro-optical response of the ChLC.

of external field $\left(U_{\text {pause }}=0 \mathrm{~V}\right)$ when the reflectance no changes more with time. Ten dependencies of the stabilized reflectance of the cholesteric display cell vs. the amplitude of the bias voltage $U_{b}$ for different values of the bias time $T_{b}(0.05 \mathrm{~s}, 0.1 \mathrm{~s}, 0.2 \mathrm{~s}, 0.3 \mathrm{~s}, 0.5 \mathrm{~s}, 0.7 \mathrm{~s}, 1 \mathrm{~s}$, $3 \mathrm{~s}, 5 \mathrm{~s}$ and $10 \mathrm{~s}$ ) have been measured. On Fig. 2 five once from these dependencies are presented. Further possibilities of analytical approximation of these dependencies will be discussed.

\section{Approximation method}

The stable texture of a ChLC in display cell is a complicated domain structure with different preferred direction of orientation of helical axes in each domain. The stabilized reflectance of a display cell depends on a distribution of such domains relative to the cell surface normal direction. Determination of the real domain distribution is enough difficult problem. Let's consider a simple model of stable cholesteric texture. In this model we have assumed that the stable cholesteric texture consists of the two type of domains: the "planar" domains where helical axes are strongly parallel and the "focal conic" domains where helical axes are strongly perpendicular to the display cell surface normal direction. It is possible to define the parameter $v$ as the part of the "planar" domains in a stable texture of a ChLC $(0 \leq v \leq 1)$. So the reflectivity $(R)$ of a stable texture of a ChLC is determined through the parameter $v$ in the following equation:

$$
R(v)=R_{\min }+\left(R_{\max }-R_{\min }\right) \times v
$$

where $R_{\min }$ and $R_{\max }$ are reflectivity of the focal conic and the planar textures correspondingly. However an approximation of electro-optical characteristics of a ChLC by formula (1) is very simplified. Therefore on the basis of behavior of curves in a Fig. 2, we propose the following dependence $R\left(T_{b}, v\right)$ that afterwards has given good concurrence of experimental and theoretical curves:

$$
R\left(T_{b}, v\right)=R_{\min }\left(T_{b}\right)+\left(R_{\max }-R_{\min }\left(T_{b}\right)\right) \times \sin \left(\frac{\pi}{2} \times v\right)
$$




\section{A. Rybalochka et al.: Approximation of electro-optical characteristics ...}

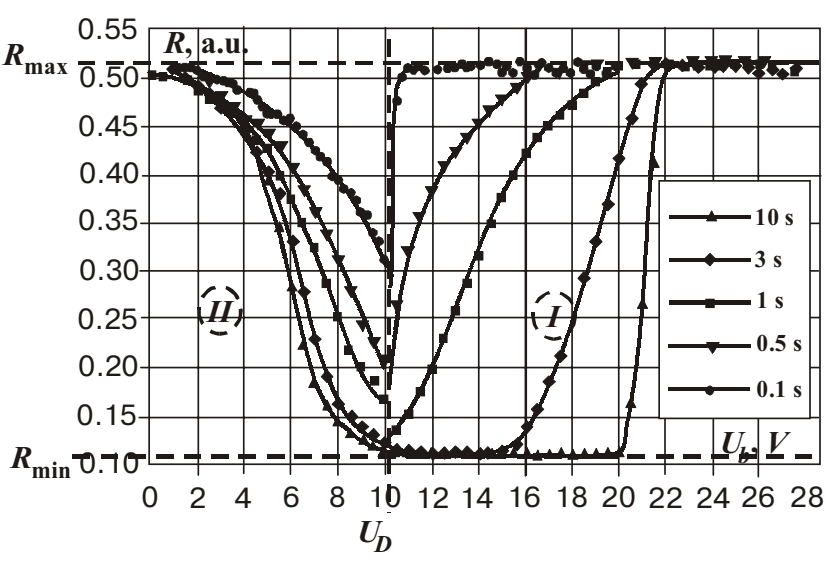

Fig. 2. Experimental dependencies $R\left(U_{b}\right)$ at different values of the bias time $T_{b}$.

where $R_{\min }\left(T_{b}\right)$ is a dependence of a minimal reflectivity of the focal conic texture vs. the time parameter $T_{b}$ of the voltage waveform on Fig. 1. The dependence $R_{\min }\left(T_{b}\right)$ is shown on Fig. 3. The value of $R_{\min }$ since value of $T_{b}=$ $=T_{F C}$ (see Fig. 3) begins to increase up to $R_{\max }$ at $T_{b} \rightarrow 0$. The physical sense of the parameter $T_{F C}$ can be formulated as follows: $T_{F C}$-is the minimal necessary duration of the voltage pulse that can address a ChLC in a display cell from the field induced homeotropic texture to the stable focal conic texture with the minimal values of $R_{\min }$. From Fig. 3 we can see that the point on this curve at $T_{b}$ $=T_{F C}$-is the point of a change of distinctive behavior of the curve $R_{\min }\left(T_{b}\right)$. At $T_{b}>T_{F C}$ the value of $R_{\min }\left(T_{b}\right)$ practically does not vary, and at $T_{b}<T_{F C}$ the value of $R_{\min }\left(T_{b}\right)$ sharply increase up to $R_{\max }$ at $T_{b}=0$. Each experimental curve on Fig. 2 and our model distribution of the "planar" and the "focal conic" domains can be presented as it is shown on Fig. 4 and 5 (Fig. 4 - for curves measured at $T_{b}>T_{F C}$, Fig. 5 - for curves measured at $T_{b}<T_{F C}$ ). For each of the curves on Figs 4 and 5 four threshold voltage can be determined: $U_{h}^{c}, U_{h-f_{c}} \max$, $U_{h-f c}{ }^{\min }$ and $U_{h-p}$. The voltage level $U_{h}^{c}$ is the minimal

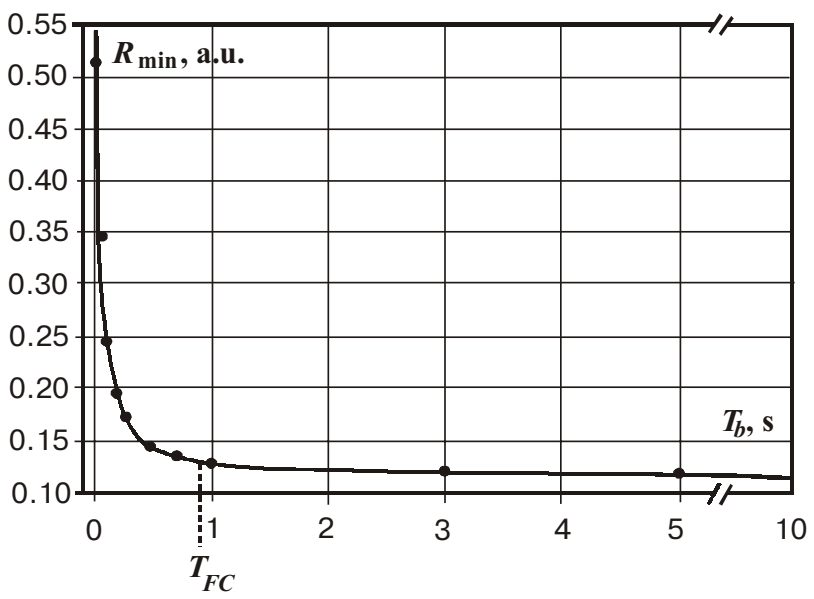

Fig. 3. The dependence $R_{\min }\left(T_{b}\right)$.

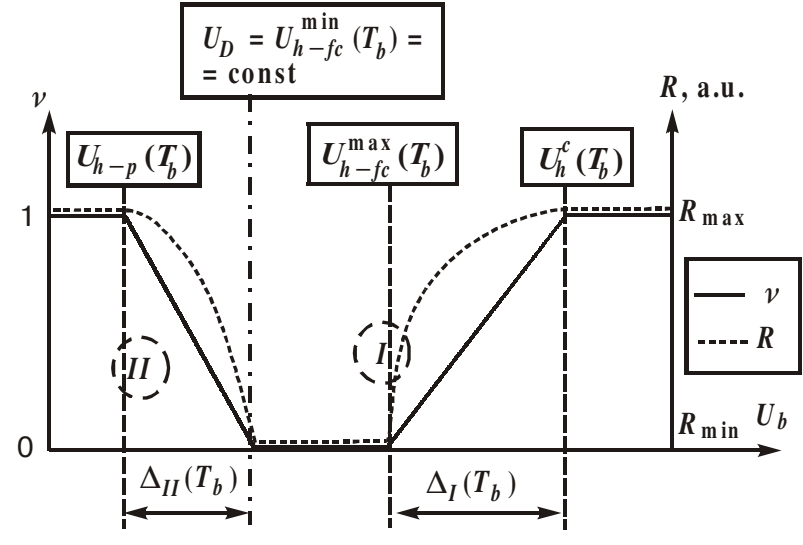

Fig. 4. Schematic form of experimental curves and model distribution of domains at $T_{b}>T_{F C}$.

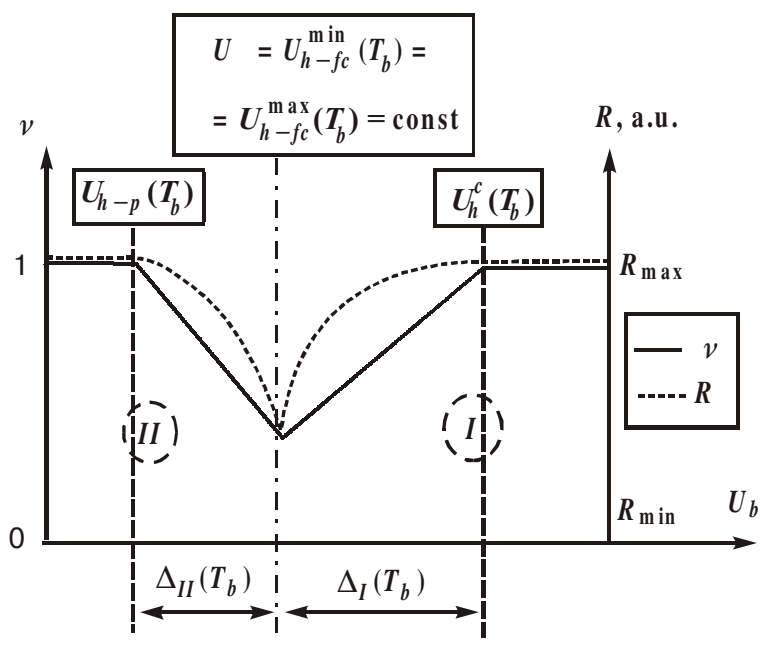

Fig. 5. Schematic form of experimental curves and model distribution of domains at $T_{b}>T_{F C}$.

voltage level that holds the field induced homeotropic texture of a ChLC in the display cell during the bias time $T_{b}$. The voltage levels $U_{h-f c} \max$ and $U_{h-f c}^{\min }$-are the maximal and the minimal voltage levels that address the cholesteric material from the field induced homeotropic texture into the final stable focal conic texture with the minimal reflectivity. And the voltage level $U_{h-p}$ is the maximal voltage level that allow to a ChLC transforms from the homeotropic texture to the stable planar texture with the maximal reflectivity.

There are two ranges of changing of the voltage level $U_{b}$ where the electro-optical curves on Fig. 2 have distinctive behavior: the first range " $P$ " - is $U_{b}>U_{D}$ and the second range " $I I$ " - is $0<U_{b}<U_{D}$ (see Figs 2, 4 and 5), where the voltage level $U_{D}$ can be determined as the minimal amplitude of a voltage pulse that can address a ChLC from the homeotropic texture to the stable focal conic texture with the minimal reflectivity. For all curves on 


\section{A. Rybalochka et al.: Approximation of electro-optical characteristics ...}

Fig. 2 in both ranges there are stable textures of a ChLC with the same reflectivity from $R_{\min }\left(T_{b}\right)$ to $R_{\max }$. These textures in our model have the same domain distribution and differ only in a sequence of textural transitions of a ChLC during applying of the voltage waveform from Fig. 1 to the display cell. In the first range " $I$ ", the planar texture in the "planar" domains is formed after the end of the action of the bias voltage. In these domains at the end of the bias time a ChLC in a display cell is in the homeotropic texture and after removal of the bias voltage it transforms through the transient planar texture to the stable planar texture. In the "focal conic" domains a ChLC begins to transform into the focal conic texture during the bias time and after removal of the bias voltage the stable focal conic texture are formed. In the second range "II", the planar texture in the "planar" domains begins to form at once after the end of the preparation time. The transition of a ChLC from the homeotropic texture to the transient planar texture and then to the stable planar texture begins already during the bias time. In those domains where a presence of the bias voltage pulse prevents the transition of a ChLC from the homeotropic texture to the transient planar texture the final focal conic texture is formed.

As we can see from formula (2) for the approximation of electro-optical characteristics of a ChLC it is necessary to set the value of the maximal reflectivity $R_{\max }$, the dependence of the minimal reflectivity vs. the bias time $R_{\min }\left(T_{b}\right)$ and to lay down the law of a change of the parameter $v$ as a function from two variables (first - is the bias voltage $U_{b}$ and the second - is the bias time $T_{b}$ ): $v=F\left(U_{b}, T_{b}\right)$. The maximal reflectivity $R_{\max }$ and the dependence of the minimal reflectivity vs. the bias time $R_{\min }$ $\left(T_{b}\right)$ can be easy measured. In our case $R_{\max }=0.51$ and the dependence $R_{\min }\left(T_{b}\right)$ is showing on Fig. 3. Ways of determination of the function $v=F\left(U_{b}, T_{b}\right)$ for the range " $I$ " $\left(v_{I}\right)$ and for the range " $I I$ " $\left(v_{I I}\right)$ will be discussed below.

In the range " $I$ " the parameter $v_{I}$ is determined in the following:

$$
v_{I}\left(U_{b}, T_{b}\right)=\left\{\begin{array}{c}
0, U_{b} \leq U_{h-f_{c}}^{\max }\left(T_{b}\right) \\
\frac{U_{b}-U_{h-f c}^{\max }\left(T_{b}\right)}{\Delta_{I}\left(T_{b}\right)}, U_{h-f_{c}}^{\max }\left(T_{b}\right) \leq U_{b} \leq U_{h}^{c}\left(T_{b}\right) \\
1, U_{b} \geq U_{h}^{c}\left(T_{b}\right)
\end{array}\right.
$$

where $\Delta_{I}\left(T_{b}\right)=U_{h}^{c}\left(T_{b}\right)-U_{h-f c}^{\max }\left(T_{b}\right)$. As we can see from formula (3) for definition of the parameter $v_{I}$ at any values of $U_{b}$, and $T_{b}$ it is necessary to lay down laws of a change of functions $U_{h}^{c}\left(T_{b}\right)$ and $U_{h-f_{c}}^{\max }\left(T_{b}\right)$ or $U_{h}^{c}\left(T_{b}\right)$ and $\Delta_{I}\left(T_{b}\right)$. On Fig. 6 dependencies $U_{h}^{c}\left(T_{b}\right), U_{h-f c}^{\max }\left(T_{b}\right)$ and $\Delta_{I}\left(T_{b}\right)$ that have been obtained from experimental curves are presented. As we can see from Fig. 6 at the $T_{b}>T_{F C}$ the value of the voltage level $U_{h}^{c}$ practically does not change and can be approximated by linear dependence with the small constant of proportionality. At

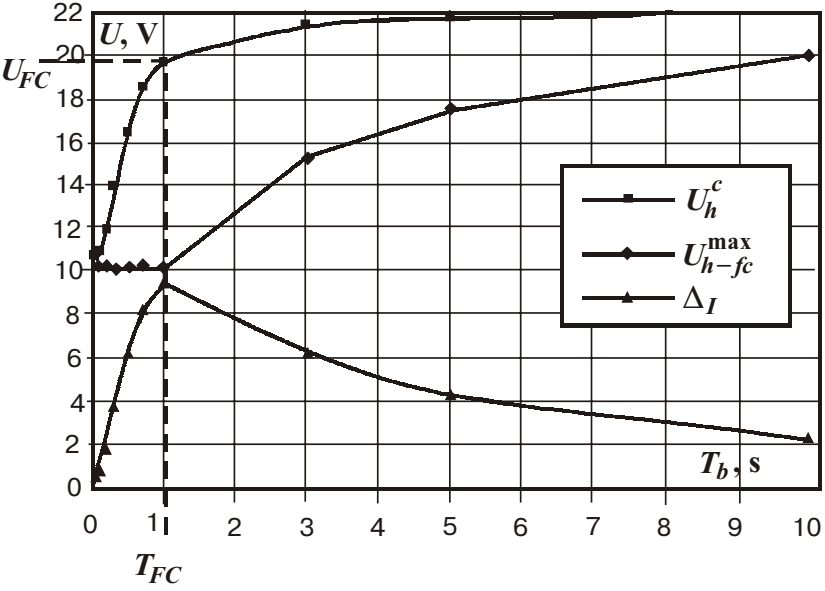

Fig. 6. Experimental dependencies $U_{h}^{c}\left(T_{b}\right), U_{h-f c}^{\max }\left(T_{b}\right)$ and $\Delta_{I}\left(T_{b}\right)$.

the $0<T_{b}<T_{F C}$ the change of the function $U_{h}^{c}\left(T_{b}\right)$ have explicitly nonlinear character. Such behavior of the function $U_{h}^{c}\left(T_{b}\right)$ can be mathematically approximated in the following:

$$
U_{h}^{c}\left(T_{b}\right)=U_{h}^{c(S T)}-\frac{U_{h}^{c(S T)}-U_{D}}{1+a_{1} \times \ln \left[1+\left(\frac{T_{b}}{T_{F C}}\right)^{2}\right]}
$$

where $a_{1}$ - is a some coefficient and $U_{h}^{c(S T)}=U_{h}^{c}(\infty)$. The coefficient $a_{1}$ probably can be determined by the physical parameters of a cholesteric mixture. But in this paper this possibility is not considered. Here we determine this coefficient directly from formula (4) using experimental data from Fig. 6 (curve $U_{h}{ }^{c}$ ). We suppose that $U_{h}^{c(S T)}=U_{h}^{c}(10 \mathrm{~s})=22.0 \mathrm{~V}$. From Fig. 2 we determine the value of the voltage level $U_{D}=10.25 \mathrm{~V}$. Also from Fig. 6 we determine the value of the voltage level $U_{h}^{c}\left(T_{F C}\right)=19.75 \mathrm{~V}$. Substituting these data in the formula (4) we determine the value of the coefficient $a_{1}=$ $=6.1$. On Fig. 7 the experimental and approximated by formula (4) dependencies of the voltage level $U_{h}^{c}$ vs. the bias time $T_{b}$ are presented. The dependence $U_{h-f c}^{\max }\left(T_{b}\right)$ can be approximated by the next way:

$U_{h-f_{c}}^{\max }\left(T_{b}\right)=\left\{\begin{array}{l}U_{D}, T_{b} \leq T_{F C} \\ U_{D}+a_{2} \times \ln \left(\frac{T_{b}}{T_{F C}}\right) T_{b} \geq T_{F C}\end{array}\right.$

where $a_{2}$-is a some coefficient. The coefficient $a_{2}$ as well as the coefficient $a_{1}$ can be determined directly from the formula (5). Substituting to this formula the value of the voltage level $U_{h-f c}^{\max }(3 \mathrm{~s})=15.25 \mathrm{~V}$ at $T_{b}=3 \mathrm{~s}$ we determine the value of the coefficient $a_{2}=4.5$. On Fig. 8 the experimental and approximated by formula (5) depend- 


\section{A. Rybalochka et al.: Approximation of electro-optical characteristics ...}

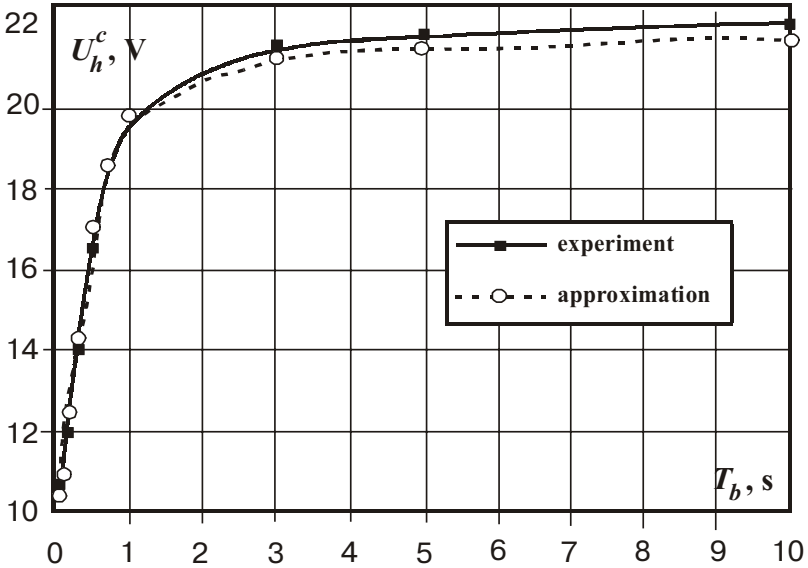

Fig. 7. Experimental and approximated dependencies the voltage level $U_{h}^{c}$ vs. the bias time $T_{b}$.

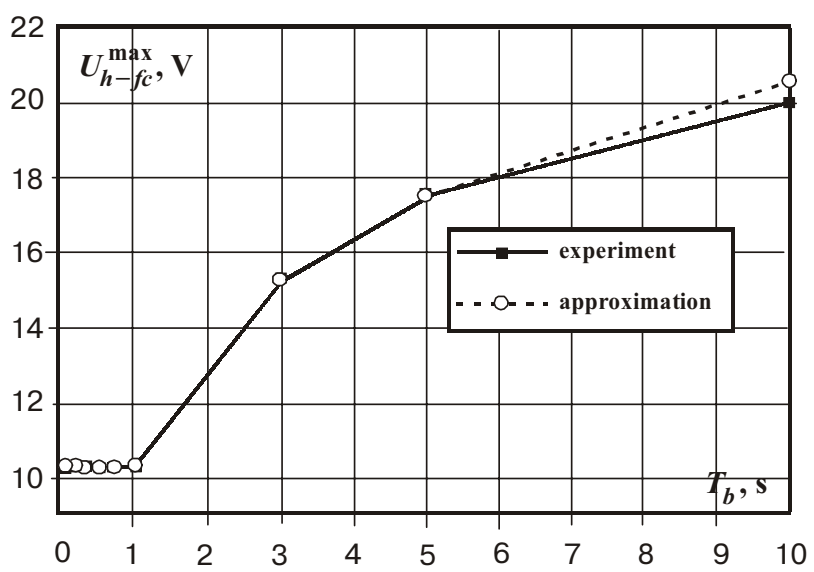

Fig. 8. Experimental and approximated dependencies the voltage level $U_{h-f c}^{\max }$ vs. the bias time $T_{b}$.

encies of the voltage level $U_{h-f_{c}}^{\max }$ vs. the bias time $T_{b}$ are presented. The experimental and approximated dependencies of the parameter $\Delta_{I}\left(T_{b}\right)=U_{h}^{c}\left(T_{b}\right)-U_{h-f_{c}}^{\max }\left(T_{b}\right)$ are presented on Fig. 9. Substituting approximated dependencies $\Delta_{I}\left(T_{b}\right)$ and $U_{h-f_{c}}^{\max }\left(T_{b}\right)$ in the formula (3) we obtain the final equation for the parameter $v_{I}\left(U_{b}, T_{b}\right)$. Using this equation and the formula (2) approximated electro-optical characteristics of the ChLC in the range " $I$ " can be obtained.

In the range " $I I$ " the parameter $v_{I I}$ is determined in the following:

$v_{I I}\left(U_{b}, T_{b}\right)=\left\{\begin{array}{c}1, U_{b} \leq U_{h-p}\left(T_{b}\right) \\ \frac{U_{D}-U_{b}}{\Delta_{I I}\left(T_{b}\right)}, U_{h-p}\left(T_{b}\right) \leq U_{b} \leq U_{D}\end{array}\right.$

where $\Delta_{I I}\left(T_{b}\right)=U_{h-f_{c}}^{\max }\left(T_{b}\right)-U_{h-p}\left(T_{b}\right)=U_{D}-U_{h-p}\left(T_{b}\right)$. It is possible to suppose that $U_{h-p}\left(T_{b}\right)=$ const $=1.5 \mathrm{~V}$ (see Fig. 2). It means that $\Delta_{I I}\left(T_{b}\right)=$ const $=8.75 \mathrm{~V}$.

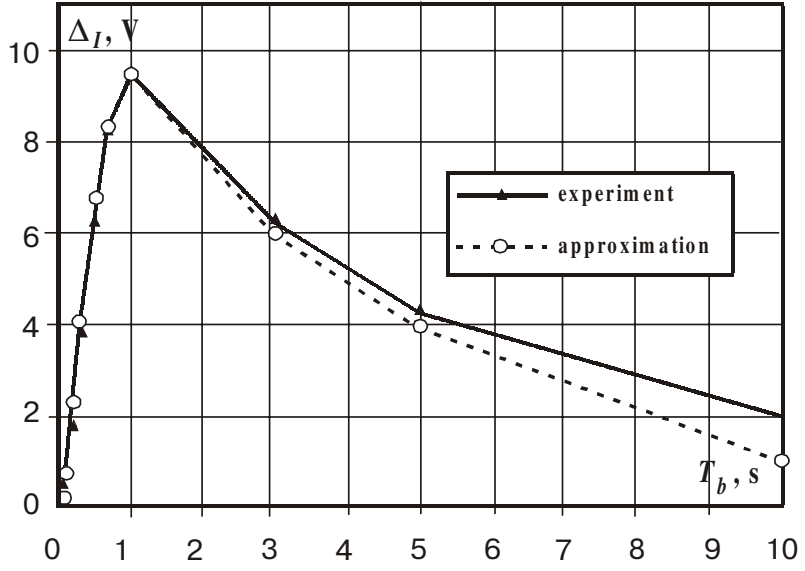

Fig. 9. Experimental and approximated dependencies the parameter $\Delta_{I}$ vs. the bias time $T_{b}$.

Substituting values of the parameter $\Delta_{I I}$ and the voltage levels $U_{D}$ and $U_{h-p}$ to the formula (6) we obtain the final equation for the parameter $v_{I I}\left(U_{b}, T_{b}\right)$. Using this equation and the formula (2) approximated electro-optical characteristics of the ChLC in the range "II" can be obtained. The final approximated dependencies $R\left(U_{b}\right)$ at different values of the bias time $T_{b}$ are presented on Fig. 10.

Comparison of electro-optical characteristics on Figs 2 and 10 shows that proposed method for an approximation of electro-optical characteristics of a ChLC gives good coincidence of experimental and approximated dependencies. Some difference in experimental and approximated dependencies is explained by simplicity of presented method. For more correct coincidence the more complex model dependence of the reflectivity vs. the parameter $v$ is needed. At the same time the curves on Figs 7, 8 and 9 demonstrate the successful selection of approximated functions for threshold voltage dependencies $U_{h}^{c}\left(T_{b}\right)$ and $U_{h-f c}^{\max }\left(T_{b}\right)$. And now, the finding of the

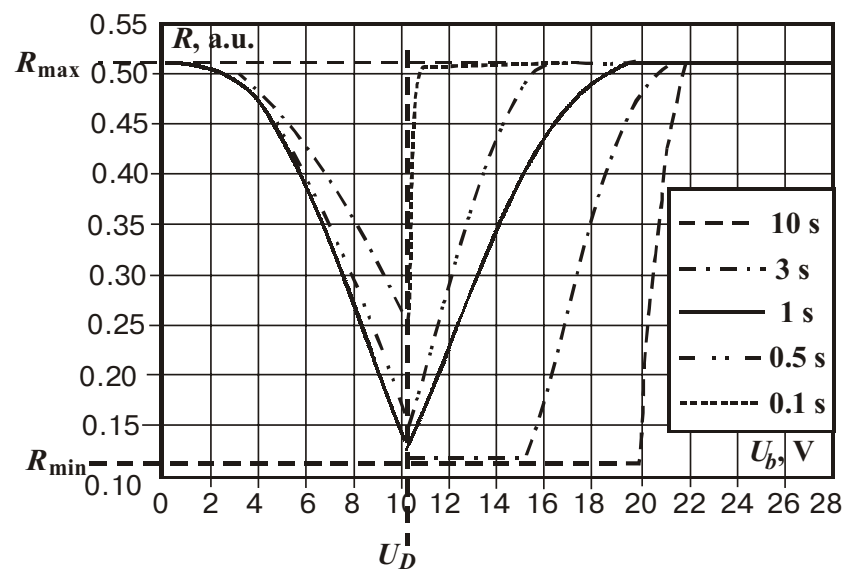

Fig. 10. Approximated dependencies $R\left(U_{b}\right)$ at different values of the bias time $T_{b}$. 


\section{A. Rybalochka et al.: Approximation of electro-optical characteristics ...}

relationship between physical properties of a ChLC and the value of coefficients $a_{1}$ and $a_{2}$ is a very important problem.

Based on our investigation we propose the next procedure for experimental study of a ChLC for an approximation of its electro-optical characteristics:

1. The measuring of the quasi-static electro-optical response of a cholesteric display cell. In this case the sequence of voltage pulses with the voltage waveform from Fig. 1 and $T_{b}>10 \mathrm{~s}$ is used. As a result we obtain values of the next parameters: $R_{\min }^{(S T)}=R_{\min }(\infty), R_{\max }, U_{h}^{c(S T)}$, $U_{h-f c}^{\max (S T)}, U_{h-p}$ and $U_{D}$.

${ }^{h}{ }_{2}^{f c}$. The measuring of the dependence $R_{\min }\left(T_{b}\right)$. For this measuring also the sequence of voltage pulses with the voltage waveform from Fig. 1 is used. But in this case the bias voltage level is fixed $\left(U_{b}=U_{D}\right)$ and the bias time is changed. As a result we obtain the experimental dependence $R_{\min }\left(T_{b}\right)$ and the value of the parameter $T_{F C}$.

3. The measuring of the electro-optical response of a cholesteric display cell as in point 1 with $T_{b}=T_{F C}$. This dependence is necessary to determine the value of the coefficient $a_{1}$.

4. The measuring of the electro-optical response of a cholesteric display cell as in point 1 with $T_{b}>T_{F C}$. This dependence is necessary to determine the value of the coefficient $a_{2}$.

The duration of experimental measuring of these four characteristics is about one hour that is on the order less than duration of experimental study necessary for investigation of electro-optical properties of a ChLC by a standard way. Moreover, using proposed method of approximation, it is possible to obtain approximated electrooptical characteristics of a ChLC, similar to characteristics on a Fig. 10, for any value of the parameter $T_{b}$.

\section{Conclusions}

In this paper the method for an approximation of electrooptical characteristics of a ChLC, which describe a behavior of a cholesteric material at transitions from the field induced homeotropic texture to the stable planar and focal conic textures has been demonstrated. This method allows to obtain sufficient quantity of approximated characteristics of a ChLC only due to the measuring of four special electro-optical characteristics. It allows to essentially decrease the duration of experimental study in comparison with then standard way of investigation.

\section{Acknowledgements}

The authors would like to gratitude Mr. P. Titarenko and $\mathrm{Mr}$. Yu. Kolomzarov for their help in preparation of liquid crystal display cells. This work was supported by STCU under the project No. 2025.

\section{References}

1. D.K. Yang, J.L. West, L.-C. Chien and J.W. Doane, Control of Reflectivity and Bistability in Displays Using Cholesteric Liquid Crystals // J. Appl. Phys., 76 (2), pp. 1331-1333 (1994).

2. X.Y. Huang, J.W. Doane, D.K. Yang, The Transient Response and Dynamic Drive of Cholesteric Liquid Crystal Displays // Journal of the SID, 5/3, pp. 179-187 (1997).

3. D.K. Yang, Z.J. Lu, Switching Mechanism of Bistable Reflective Cholesteric Displays // SID 95 Digest, pp.351-354 (1995).

4. X.Y. Huang, D.K. Yang, P. Bos and J.W. Doane, Dynamic Drive for Bistable Reflective Cholesteric Displays: A Rapid Addressing Scheme // SID 95 Digest, pp.347-350 (1995).

5. M. Zhu, D.K. Yang, High-Speed Dynamic Drive Scheme for Bistable Reflective Cholesteric Displays // SID 97 Digest, pp. 97-100 (1997).

6. A. Kozachenko, P. Oleksenko, V. Sorokin, V. Nazarenko, Histeresis as a Key Factor for the Fast Control of Reflectivity in Cholesteric LCDs // Conference Record of the IDRC 97, p.148-151 (1997)

7. A. Rybalochka, V. Sorokin, S. Valyukh, A. Sorokin, V. Nazarenko, Dynamic Drive Scheme for Fast Addressing of Cholesteric Displays // SID 2000 Digest, pp. 818-821 (2000). 